\title{
The propagation of UHE cosmic rays
}

\author{
Martin Pohl ${ }^{\star a b}$ \\ ${ }^{a}$ Institut für Physik und Astronomie, Universität Potsdam, 14476 Potsdam-Golm, Germany \\ ${ }^{b}$ DESY, 15738 Zeuthen, Germany \\ E-mail: pohlmadq@gmail.com
}

\section{David Eichler}

Physics Department, Ben-Gurion University, Be'er-Sheva 84105, Israel

E-mail: eichler.david@gmail.com

\begin{abstract}
We study the propagation of ultra-high-energy cosmic rays (UHECR) in the Galaxy, concentrating on the energy range below the ankle. The particles above the ankle are generally believed to be extragalactic, showing both a flattening of slope in the observed flux, and little anisotropy below the GZK cutoff, and some anisotropy towards the local supercluster above it. The particles below the ankle could well be galactic in origin, but the viability of this scenario has not been convincingly demonstrated to date. In this paper, we consider the hypothesis that UHECR production scales with star formation, which includes the hypothesis of UHECR origin from long GRB [13]. In an earlier publication we have already demonstrated that forward shocks from long GRB in the interstellar medium are energetically sufficient to produce the Galactic cosmic-ray component up to the ankle at $4 \times 10^{18} \mathrm{eV}$. Here we investigate in detail other observational constraints. A Monte-Carlo method based on analytical solutions to the time-dependent diffusion problem is used to account for intermittency. Assuming a source population similar to that of long GRB, we derive constraints arising from intermittency and the requirement to satisfy observational limits on the composition and anisotropy. It is shown that the composition and anisotropy at $10^{18} \mathrm{eV}$ are difficult to reproduce and require that either the particle mean free path is unusually small or that the composition is heavier than suggested by recent Auger data. We therefore consider it highly desirable that steps be taken to reduce the systematic uncertainty in the experimental derivation of the UHECR composition around $10^{18} \mathrm{eV}$.
\end{abstract}

25th Texas Symposium on Relativistic Astrophysics - TEXAS 2010

December 06-10, 2010

Heidelberg, Germany

* Speaker. 


\section{Introduction}

An open problem in cosmic-ray astrophysics is at what energy we observe the transition from a Galactic to an extragalactic origin of particles. A closely related question is which sources in the Galaxy contribute ultra-high energy cosmic rays (UHECR). The limit on inferred source power per unit baryon mass required to sustain Galactic UHECR in the [4-40] EeV range that is imposed by the observed anisotropy limits is smaller by nearly 3 orders of magnitude than what is required for an extragalactic origin, as calculated in [9], and it corresponds to the power per unit mass of gamma rays from GRB [10]. This numerical coincidence fits the hypothesis of a GRB origin for the Galactic component of UHECR, without invoking a much larger unseen energy reservoir for GRB. In fact, it would allow a Galactic origin for UHECR above the ankle were it somehow possible to trap these CR within the Galaxy effectively enough to obey the isotropy constraint. It remains to be shown that applying the hypothesis of UHECR from Galactic GRB to subankle Galactic CR, for which there is no extra-Galactic alternative, obeys the isotropy constraint, and this analysis is done in this paper.

We study the time-dependent diffusive transport of UHECR in the Galaxy using the method of Monte-Carlo to account for the unknown location and explosion time of GRB or other sources with similar population statistic. This approach permits us to accurately account for intermittency effects in the local UHECR spectrum and thus goes beyond the scope of earlier publications $[13,17,8]$.

We assume the propagation in the Galaxy of cosmic rays at energies $10^{15} \mathrm{eV}$ to $10^{18} \mathrm{eV}$ can be accurately described as isotropic diffusion. This requires, a) that the particle Larmor radii are smaller than the largest scale on which the Galactic magnetic field is turbulent, otherwise scattering is likely inefficient, and $b$ ) that the particle mean free path, $\lambda_{\operatorname{mfp}}$, is much smaller than a few kpc, the typical distance between the solar system and a GRB in the Galaxy. When condition b) does not hold, the isotropy problem derived in this paper can only be exacerbated. The Larmor radius of a $Z=1$ particle in a $10 \mu \mathrm{G}$ field reaches $\sim 100 \mathrm{pc}$ at $10^{18} \mathrm{eV}$, and therefore the first condition should hold for UHECRs of any composition below $\approx 10^{18} \mathrm{eV}$. The second condition requires that $\lambda_{\text {mfp }}$ be within a factor of $\sim 10$ of the Larmor radius. The assumption of isotropic diffusion is of course questionable if the magnetic field in the Galaxy is sufficiently ordered. For example, a nearly toroidal magnetic field can result in sub-Bohm diffusion in the radial and vertical directions and a much larger diffusion coefficient in the azimuthal direction. But, because of near azimuthal symmetry imposed by the nearly toroidal B field, this can be approximated with an averaged isotropic diffusion coefficient.

To evaluate the level of systematic uncertainties in our model description, we explore various geometric forms of the propagation volume of UHECR in the Galaxy. We find that a disk-like geometry, which appears more likely to be accurate than the assumption of spherical symmetry, renders the observational constraints on anisotropy and composition more difficult to meet.

\section{UHE Cosmic-ray propagation}

In the energy band of interest, escape is the dominant loss process of cosmic rays in the Galaxy. The halo size, $H$, is not well known. We use $H=5 \mathrm{kpc}$, which is at the high end of the range of likely values. We thus probably underestimate the flux suppression arising from a finite halo size; 
consequently our results on spectral structure and anisotropy are conservative for the arguments that follow. Instead of a computationally expensive full solution of the diffusion problem in disk geometry [7], we use a steady-state solution to rewrite the propagation equation in terms of the midplane cosmic-ray density, $N_{0}$, as well as turn the diffusive flux at the halo boundaries $(z= \pm H)$ into a simple catastrophic loss term,

$$
\tau_{\mathrm{esc}}=\frac{H^{2}}{2 D} \simeq\left(1.2 \cdot 10^{6} \mathrm{yr}\right)\left(\frac{H}{5 \mathrm{kpc}}\right)^{2}\left(\frac{\lambda_{\mathrm{mfp}}}{0.1 \mathrm{kpc}}\right)^{-1} .
$$

Ignoring variations in the diffusion coefficient within the Galactic plane, the problem depends only on the in-plane distance between source (GRB) and observer, $\rho$, and can be recast as 2-D diffusion equation for the mid-plane cosmic-ray density around a point source,

$$
\frac{\partial N_{0}}{\partial t}+\frac{N_{0}}{\tau_{\mathrm{esc}}}-\frac{1}{\rho} \frac{\partial}{\partial \rho}\left(\rho D \frac{\partial N_{0}}{\partial \rho}\right)=Q(E) \delta(t) \frac{\delta(\rho)}{2 \pi \rho H}
$$

whose solution is

$$
N_{0}(\rho, t, E)=\exp \left(-\frac{t}{\tau_{\mathrm{esc}}}\right) \frac{\Theta(t)}{4 \pi D t} \frac{Q(E)}{H} \exp \left(-\frac{\rho^{2}}{4 D t}\right) .
$$

The anisotropy in the case of a single GRB is

$$
\delta \simeq \lambda_{\operatorname{mfp}} \frac{1}{N_{0}}\left|\vec{\nabla} N_{0}\right| \simeq \frac{3 \rho}{2 c t}
$$

Heavier nuclei have a smaller rigidity at the same total energy, $R \propto E / Z$. The mean free path of an ultra-high-energy particle should only depend on the rigidity, and in the absence of energy losses a nucleus of charge $Z$ and energy $E_{Z}$ should behave like a proton of energy $E=E_{Z} / Z$. Thus equation 2.3 also describes the distribution of heavy nuclei in the Galaxy, provided the appropriate scaling is applied to the energy and the source rate.

Generally, GRBs in the Galaxy are expected every million years or so, the exact rate depending on the beaming fraction and the detailed scaling of long GRB with star formation and metallicity (For a detailed review see [11]). Therefore, only a small number of GRB can contribute to the particle flux at the solar circle, and their relative contribution depends on the location and explosion time of the GRB. Variations in the local particle flux must be expected, and neither the particle spectrum from an individual GRB nor the spectrum calculated for a homogeneous source distribution are good proxies. To fully account for discreteness of GRBs in space and time, we can use the method of Monte-Carlo to randomly place GRBs in the Galaxy with given spatial probability distribution in galactocentric radius

$$
P\left(r_{G C}\right)=\frac{2 r_{G C}}{r_{0}^{2}} \exp \left(-\frac{r_{G C}^{2}}{r_{0}^{2}}\right)
$$

with scale $r_{0}=5 \mathrm{kpc}$, and with given GRB rate. We have calculated spectra for $10^{4}$ random sets of GRBs, going back 6 Gyr in time, but ignoring energy losses through inelastic collisions. Overall, the method is the same as that used to model the transport of cosmic-ray electrons in the Galaxy $[16,15]$. Results are shown in Figure 1, where we have assumed an injection index $s=2$. More details are found in an upcoming publication (Pohl \& Eichler, in prep.). 


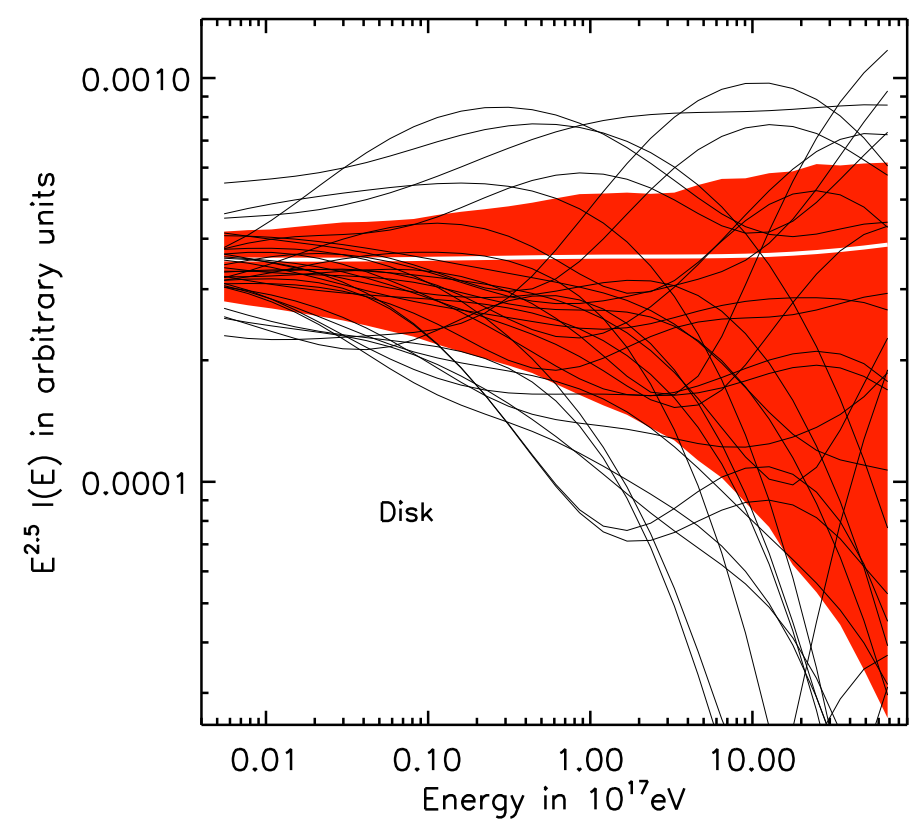

Figure 1: Proton spectra at the solar circle expected for a diffusion coefficient scaling with $\sqrt{E}$ in disk geometry. The red band indicates the central $68 \%$ containment region for the particle flux at the given energy.The GRB rate is set to $5 \mathrm{Myr}^{-1}$. We have plotted 31 individual, randomly selected spectra. The average spectrum is given by the thick white line.

It is the energy dependence of the diffusion coefficient that determines the particle spectrum. Structure in the observed spectrum could thus arise from changes in the energy dependence, e.g. from shallow at lower energies to Bohmian at higher energies, without requiring any structure in the source spectrum (see also Calvez et al. 2010). Intermittency is strong for a GRB rate below 1 per Myr, in particular for the more realistic disk geometry. In essence, the local UHECR spectrum from galactic GRBs is unpredictable if the scattering mean free path exceeds about $100 \mathrm{pc}$, which for the parameters used here is the case above $10^{17} \mathrm{eV}$ for protons, and above $3 \cdot 10^{18} \mathrm{eV}$ for iron. Model fits of single-source spectra can thus be very misleading [17]. The actually expected spectra display bumps unrelated to both source and propagation physics, some of which may indeed be observed [5]. The absence of very large bumps in the observed UHECR spectra suggests that either the mean free path for scattering is smaller than assumed here, or the rate of cosmic-ray producing GRBs in the Galaxy exceeds 1 per Myr, at which the amplitude of such bumps becomes smaller. Generally, careful accounting of the statistical fluctuations is mandatory for properly estimating the local UHECR spectrum from GRBs [8].

\section{A possible model}

We now try to construct a model that reproduces the spectrum of cosmic rays between $10^{15} \mathrm{eV}$ and $10^{19} \mathrm{eV}$ together with the anisotropy limits and the composition. We use data of the KascadeGrande collaboration [4], HiRes [1, 12], and the Auger collaboration [3, 14]. At $10^{18} \mathrm{eV}$ the anisotropy is low, $\delta \leq 0.01$ (the $99 \%$ upper limit is 0.02 ), and the composition is light, but not necessarily dominated by protons [2]. 


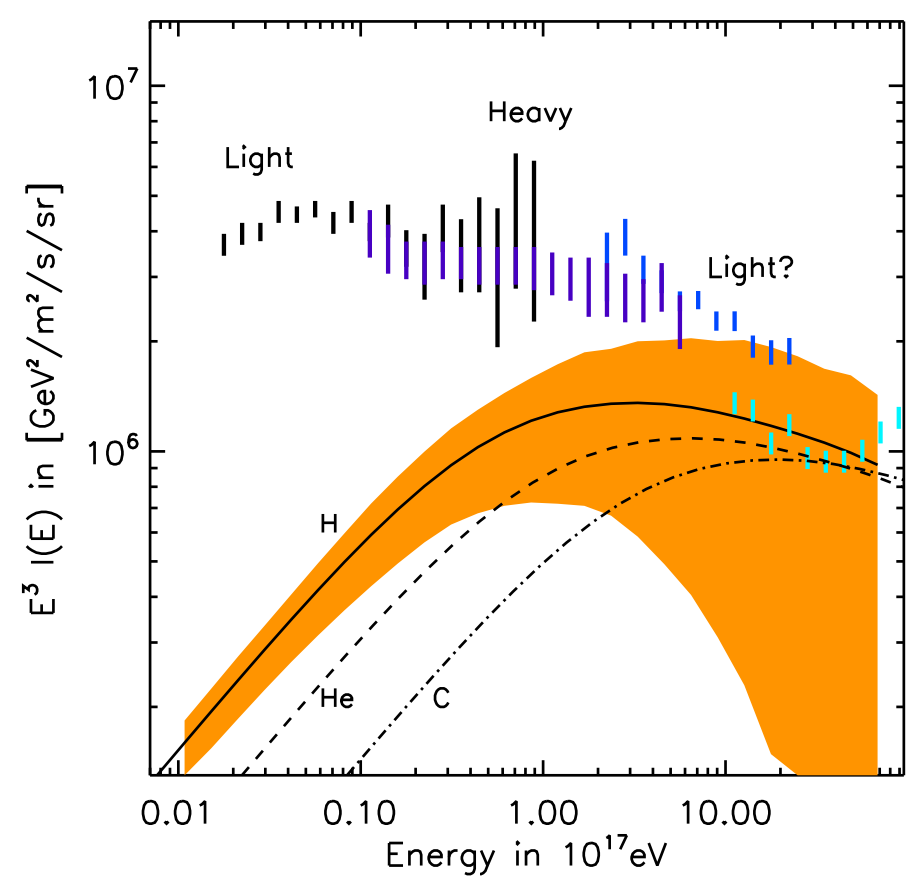

Figure 2: Model spectra for $\mathrm{H}, \mathrm{He}$, and $\mathrm{C}$ nuclei, including the $68 \%$ variation range in the case of protons. The solid line denotes the average spectrum of hydrogen, the dashed line displays the same for helium, and the dash-dotted line is for carbon. Also shown are the spectra measured with KASCADE-Grande, HiRes, and Auger, together with labels indicating the composition.

Figure 2 shows the spectra for a possible model configuration, where for simplicity we display only spectra for protons, helium, and carbon as proxies for light and heavy nuclei, respectively. The GRB rate is set to $P(t)=1 \mathrm{Myr}^{-1}$ and the source spectral index is $s=2.1$. The mean free path transitions from a shallow energy dependence to Bohmian scaling $(\propto E)$ as the particle energy increases,

$$
\lambda_{\mathrm{mfp}}=\lambda_{0} E^{0.3}\left[1+\frac{E}{60 \mathrm{PeV}}\right]^{0.7}
$$

where $\lambda_{0}=\eta r_{L}$ is chosen so a proton has a mean free path that is a certain multiple $\eta$ (unity in Figure 2) of $11 \mathrm{pc}$ at $10^{17} \mathrm{eV}$, its Larmor radius in a $10-\mu \mathrm{G}$ magnetic field. For comparison available data are displayed in the same figure. The offset between spectra from different experiments is likely due to errors in the absolute energy scale. The calculated spectrum below $10^{17} \mathrm{eV}$ is far below the data to accommodate other Galactic sources of cosmic rays, such as SNR or PWN. The fluctuation amplitude at energies above $10^{17} \mathrm{eV}$ is large for the GRB rate used here, one per Myr.

The average spectrum depends weakly on the absolute value of the mean free path at high energies. We can calculate the cosmic-ray source power required to sustain the observed flux of UHECRs at $10^{18} \mathrm{eV}$, which does depend on the mean free path. For an injection spectrum $\propto E^{-2.1}$ extending from the $\mathrm{GeV}$ band to the highest energies, fitting the observed flux of UHRCRs at $10^{18} \mathrm{eV}$ requires the source power

$$
P_{\mathrm{CR}}=P(t) \int_{1 \mathrm{GeV}} d E E Q_{0} E^{-s} \simeq \frac{\lambda_{\mathrm{mfp}}}{r_{L}}\left(10^{37} \mathrm{erg} / \mathrm{s}\right)
$$




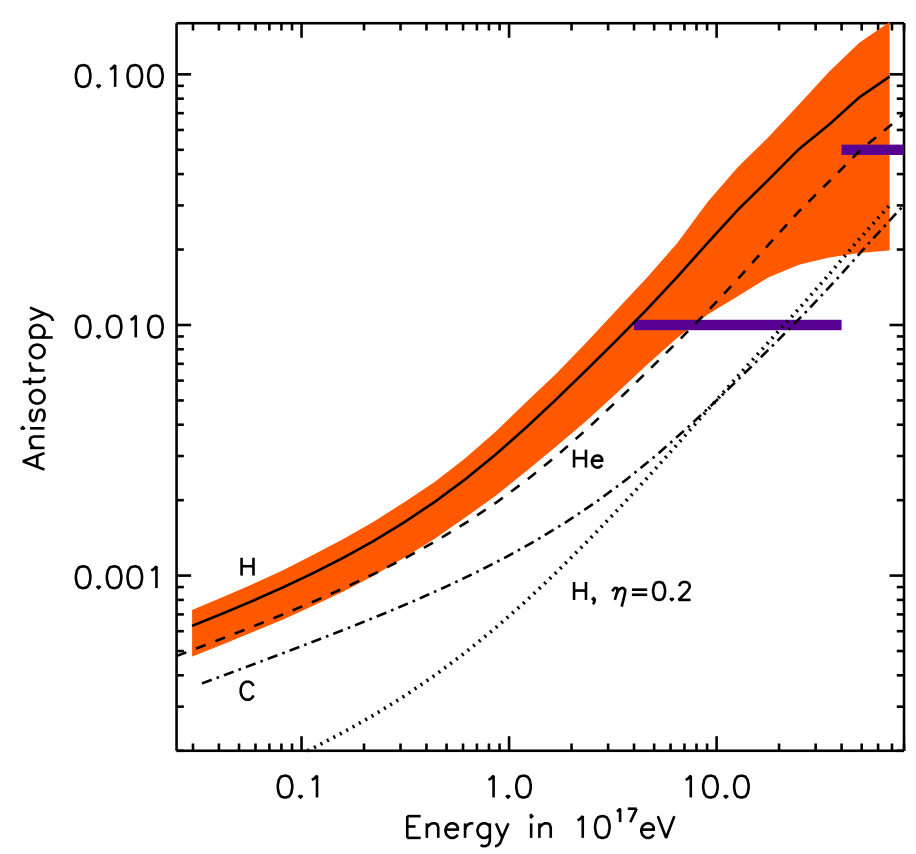

Figure 3: The expected anisotropy for different cosmic-ray nuclei, including the $68 \%$ variation range in the case of protons. The solid line denotes the average spectrum of hydrogen, the dashed line displays the same for helium, and the dash-dotted line is for carbon. For comparison, we also show the hydrogen anisotropy for a reduced mean free path, $\lambda_{\mathrm{mfp}}=0.2 r_{L}$ at high energies. The horizontal bars indicate observational upper limits.

The source power in the energy interval $\left[10^{17}, 10^{18}\right] \mathrm{eV}$ alone is

$$
P_{\mathrm{EeV}} \simeq \frac{\lambda_{\mathrm{mfp}}}{r_{L}}\left(3.5 \cdot 10^{35} \mathrm{erg} / \mathrm{s}\right)
$$

This power requirement is sufficiently low to permit viability of the GRB scenario, in contrast to the case of extragalactic UHECRs at GZK energies [9].

The anisotropy at $10^{18} \mathrm{eV}$ is not easy to keep below the upper limit established with Auger data. Figure 3 shows the anisotropy for protons, helium nuclei, and carbon for a mean free path follows equation 3.1 and $\lambda_{0}=\eta r_{L}$ chosen so that at high energy $\eta=1$ and, for hydrogen only, $\eta=0.2$, respectively. The observational upper limits are indicated by horizontal bars and reflect averages over a finite energy range that is weighted with the UHECR flux and experiment-specific efficiency function. To be noted from the figures is that the Auger limits, $\delta \leq 0.01$ at $10^{18} \mathrm{eV}$, are marginally violated by helium nuclei in the case of Bohm diffusion, and the mean free path would have to be at least a factor 5 smaller than the particle Larmor radius, if the dominant particle species were protons.

On average, Galactic long GRB need only contribute about $10^{37} \mathrm{erg} / \mathrm{s}$ in accelerated particles to fully account for the observed particle flux at $10^{18} \mathrm{eV}$, assuming a Bohmian mean free path. UHECR from Galactic long GRB can meet the observational limits on anisotropy, if the mean free path for scattering is sufficiently small. Contributing the observed sub-ankle particles (at $10^{18} \mathrm{eV}$ ) requires Bohmian diffusion if the UHECR are as heavy as carbon. A light composition such as protons or helium requires sub-Bohmian diffusion, which is a highly unlikely situation for isotropic 
diffusion, however, it could possibly be interpreted as being due to a nearly azimuthal field, with restricted cross-field diffusion. We have not investigated the effects of a Galactic guiding field that may modify the probability of escape from the Galactic disk.

Auger data suggest that at $10^{18} \mathrm{eV}$ the composition is indeed light, thus posing a problem for the notion that Galactic GRB (or any other source class with similar population statistic) produce the observed UHECR up to the ankle. This measurement is not undisputed, though, for the KASCADE-Grande collaboration has just published their analysis results which seem to favor a relatively heavy composition nearly up to $10^{18} \mathrm{eV}$ [5]. The UHECR composition is a very critical constraint, but its measurement is subject to considerable systematic uncertainties arising its dependence on models for the development of air showers. It is imperative that measures be taken to better understand the air-shower physics near $10^{18} \mathrm{eV}$.

Much of the UHECR anisotropy results from the fact that the sources are assumed to be located in the inner Galaxy, which are mostly to one side of us. As there is no power problem with Galactic GRB, it may therefore be worthwhile to consider short GRB. While they supposedly provide less power as a population than long GRB, they may have a very extended spatial distribution in the Galaxy, thus strongly reducing the expected UHECR anisotropy [6].

Support from the Israel Science Foundation, the Israel-U.S. Binational Science Foundation, and the Joan and Robert Arnow Chair of Theoretical Astrophysics is gratefully acknowledged.

\section{References}

[1] Abbasi, R., et al. 2005, Physics Letters B, 619, 271

[2] Abraham, J., et al. 2010, Physical Review Letters, 104, 091101

[3] Abraham, J., et al. 2008, Physical Review Letters, 101, 061101

[4] Apel, W. D., et al. 2009, Astroparticle Physics, 31, 86

[5] Arteaga-Velázquez, J. C., et al. 2010, arXiv:1009.4716

[6] Berger, E. 2010, ApJ, 722, 1946

[7] Büsching, I., Kopp, A., Pohl, M., Schlickeiser, R., Perrot, C., \& Grenier, I. 2005, ApJ, 619, 314

[8] Calvez, A., Kusenko, A., \& Nagataki, S. 2010, Physical Review Letters, 105, 091101

[9] Eichler, D., Guetta,D. \& Pohl, M. 2010, ApJ 722, 543

[10] Eichler, D., \& Pohl, M. 2011, ApJ submitted

[11] Gehrels, N., Ramirez-Ruiz, E., \& Fox, D. B. 2009, Ann. Rev. Astron. \& Astroph., 47, 567

[12] The High Resolution Fly'S Eye Collaboration, et al. 2009, Astroparticle Physics, 32, 53

[13] Levinson, A. \& Eichler, D. 1993, ApJ 418, L386

[14] Pierre AUGER Collaboration, et al. 2010, Physics Letters B, 685, 239

[15] Pohl, M., Perrot, C., Grenier, I., Digel, S. 2003, A\& A 409, 581

[16] Pohl, M., \& Esposito, J. A. 1998, ApJ, 507, 327

[17] Wick, S. D., Dermer, C. D., \& Atoyan, A. 2004, Astroparticle Physics, 21, 125 